\title{
Digitalización de la técnica EIS-FRA como respuesta al problema de multisensado simultáneo y portabilidad
}

\author{
Arturo Sánchez González, Nicolás Medrano, Belén Calvo \\ Grupo de Diseño Electrónico (GDE) \\ Instituto de Investigación en Ingeniería de Aragón (I3A) \\ Universidad de Zaragoza, Mariano Esquillor s/n, 50018, Zaragoza, Spain. \\ Tel. +34-976762707, e-mail: asgonzalez@unizar.es
}

\section{Resumen}

Este trabajo presenta un acercamiento íntegramente digital a la clásicamente analógica espectroscopia de impedancia electroquímica (EIS) por análisis de respuesta frecuencial $(F R A)$. El sistema diseñado se basa en un procesador multinúcleo comercial de bajo coste como único elemento activo, logrando multisensado de altas prestaciones sin comprometer consumo, portabilidad y miniaturización.

\section{Introducción}

La espectroscopia de impedancia electroquímica (EIS) es una técnica de sensado biológico en la cual la muestra bajo estudio es sometida a una excitación alterna con el fin de evaluar, en un determinado rango de frecuencias, su impedancia compleja equivalente. Esta técnica ha sufrido un gran desarrollo en la última década, presentando como principal atractivo el minimizar el riesgo de deterioro del analito y biointerfase al requerirse, comparativamente, bajos niveles de excitación $(\sim \mathrm{mV})$. Pese a ello, el número actual de diseños portables funcionales, bien sea implementados en dominio digital mediante el algoritmo de transformada rápida de Fourier (EIS$F F T$ ), bien sobre dominio analógico mediante técnicas de demodulación síncrona (EIS-FRA), es muy reducido; prácticamente inexistente si se exigen altas prestaciones o carácter multicanal [1]. La dificultad radica en ser los primeros muy exigentes a nivel de recursos hardware, $\mathrm{y}$ en requerir los segundos ineludiblemente bloques adicionales en la conformación de la cadena completa de adquisición (post-procesado, digitalización, transferencia a sistema microcontrolado/ordenador).

\section{Propuesta de EIS-FRA digital}

Como alternativa, se propone la implementación de un nuevo sistema de EIS-FRA en el cual actuación, medida, detección sensible a fase y filtrado sean íntegramente digitalizadas mediante un microcontrolador Parallax Propeller ${ }^{\circledR}$ de 8 núcleos [2] como elemento central y único activo, íntegramente programado en lenguaje ensamblador para optimizar sus prestaciones.

Así, el sistema de actuación se fundamenta en un convertidor digital-analógico $\Delta(\triangle-D A C)$ basado en la técnica de modulación por densidad de pulsos $(P D M)$. El cuantizador lo constituye el bit de desbordamiento del registro sumador de uno de los dos módulos de conteo del núcleo escogido para generación de señales; el demodulador es un filtro $\mathrm{RC}$ de segundo orden en cascada. Un diagrama de bloques del $\Delta$-DAC propuesto puede observarse en la Figura 1.a. En lo que respecta a las prestaciones logradas para el sistema de actuación, cada núcleo es capaz de generar hasta 4 señales sinusoidales simultáneamente y con desfases programables en tiempo de compilación con resolución de $0.02^{\circ}$. Con respecto a la frecuencia de las sinusoides, esta puede escogerse en el rango de $1.1 \mu \mathrm{Hz}$ a $150 \mathrm{kHz}$ mediante dos palabras digitales de 14 bits como ajuste grueso y 32 bits como ajuste fino; manteniendo un índice de distorsión armónica THD $\leq 0.73(3) \%$ y rango dinámico libre de espurios SFDR $\leq 0.61(2) \%$ en todo el ancho de banda del generador.

Siguiendo la misma filosofía, la digitalización de la señal procedente del biosensor se fundamenta en la implementación de un convertidor analógico-digital $\Sigma \Delta(\Sigma \Delta-A D C)$ mediante PDM, empleando un filtro $\mathrm{RC}$ como integrador, y como cuantizador el biestable D presente en uno de los dos módulos de conteo del núcleo escogido. El diagrama de bloques correspondiente se muestra la Figura 1.b. Por tanto, con un núcleo, el sistema es capaz de recuperar magnitud y fase en el rango frecuencial de $1.1 \mu \mathrm{Hz}$ a $10 \mathrm{kHz}$ con un número efectivo de bits, ENOB = 12 bits; aunque otras configuraciones son posibles y han sido probadas, como escoger un rango de $1.1 \mu \mathrm{Hz}$ a $40 \mathrm{kHz}$ comprometiendo un ENOB de 10 bits.

Dado que el microcontrolador dispone de 8 núcleos, este es capaz de generar hasta 32 sinusoides, recuperar hasta 32 señales arbitrarias, o cualquier 
combinación de ambas; y en lo que a EIS-FRA digital se refiere, caracterizar hasta 7 biosensores simultáneamente.

La aplicabilidad del sistema diseñado ha sido puesta a prueba en la caracterización de una membrana lipídica bicapa atada (tBLM) [3], modelizada a través de una célula de Randles [4]. Los resultados del estudio de EIS por FRA obtenidos experimentalmente y mediante simulación para el módulo y fase de la impedancia compleja equivalente de la célula se muestran en la Figura 2.

\section{Conclusiones}

En el presente trabajo se ha elaborado un estudio de las actuales técnicas de EIS existentes, se han identificado las principales dificultades en su implementación a sistemas portables y multicanal, y se ha propuesto la íntegra digitalización del método de FRA como alternativa. El planteamiento se ha llevado a término en el diseño y caracterización de un sistema de FRA-digital de hasta 7 canales, fundamentado en un microprocesador multinúcleo de bajo coste como único elemento activo. Finalmente, el sistema ha sido puesto a prueba exitosamente en la caracterización experimental de una célula de Randles como modelo eléctrico de una tBLM.

\section{REFERENCIAS}

[1]. RAIRIGH, D., MASON, A., and YANG, C. Analysis of On-Chip Impedance Spectroscopy Methodologies for Sensor Arrays. Sensor Letters. 2006, 4(4), 398402. Available from: doi:10.1166/sl.2006.054.

[2]. PARALLAX SEMICONDUCTOR. Propeller P8X32A Datasheet. Revisión 1.4. Rocklin, California, 2011.

[3]. RAGUSE, B. et al. Tethered Lipid Bilayer Membranes: Formation and Ionic Reservoir Characterization. Langmuir. 1998, 14(3), 648-659. Available from: doi:10.1021/la9711239.

[4]. RANDLES, J.E.B. Kinetics of rapid electrode reactions. Discuss. Faraday Soc. 1947, 1, 11-19. Available from: doi:10.1039/df9470100011

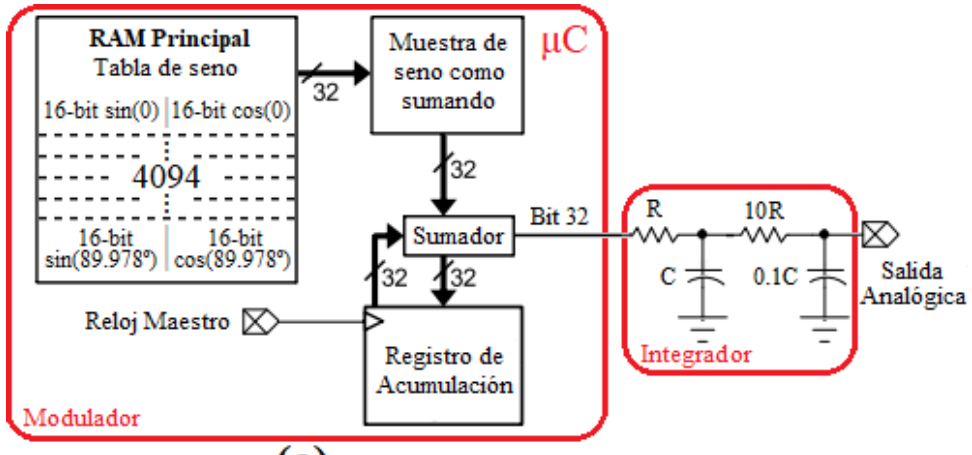

(a)

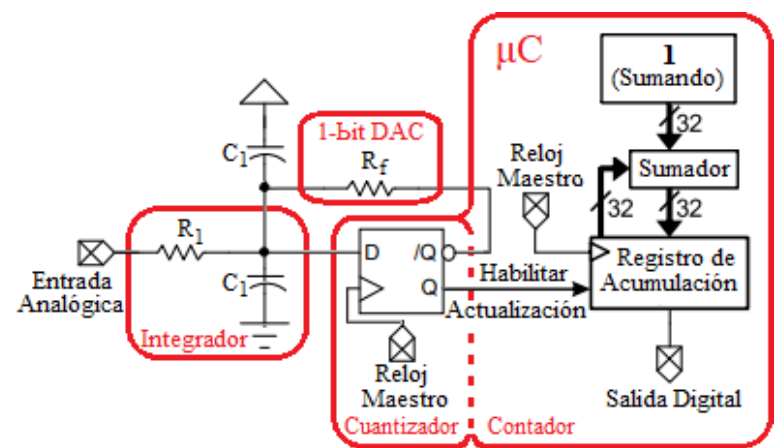

(b)

Figura 2. Sistema de actuación $\Delta$-DAC (a), y medida $\Sigma \Delta$-ADC (b), del diseño de EIS-FRA digital propuesto.
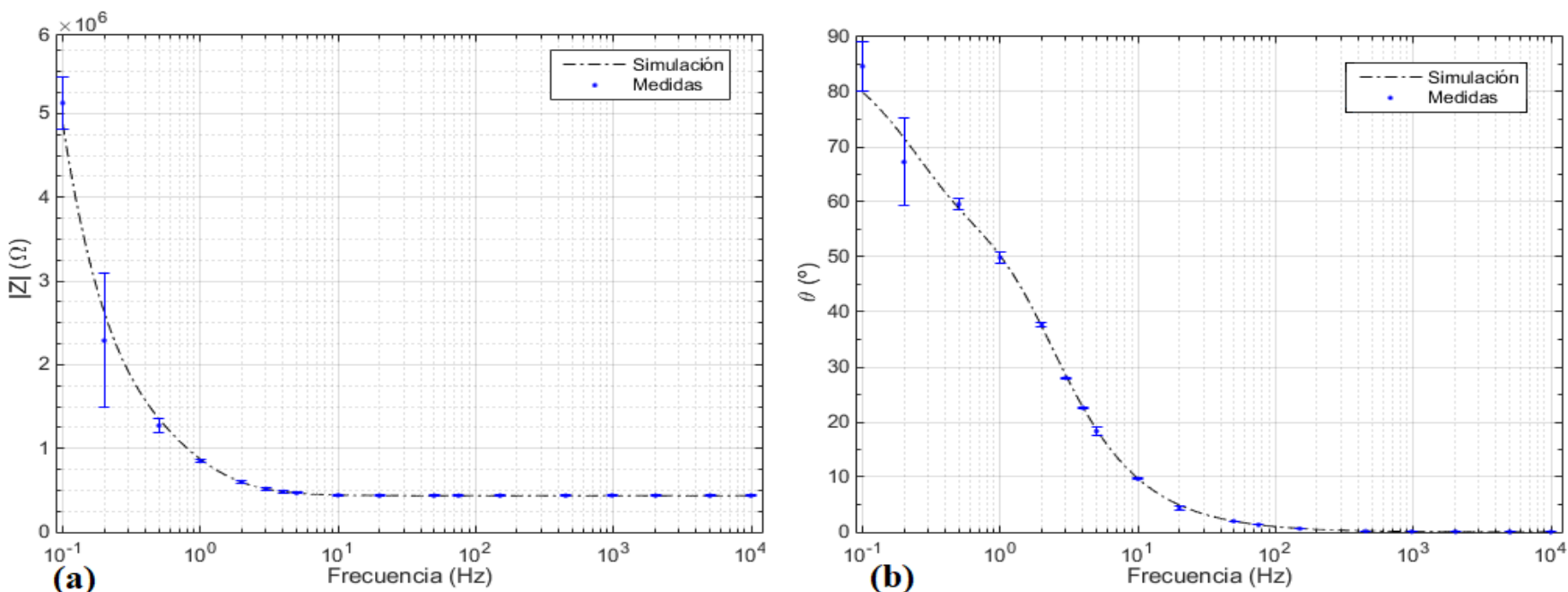

Figura 1. Magnitud (a), y fase (b), de la impedancia compleja equivalente de la célula propuesta.

Revista “Jornada de Jóvenes Investigadores del I3A”, vol. 7 (Actas de la VIII Jornada de Jóvenes Investigadores del I3A - 6 de junio de 2019). ISSN 2341-4790. 